\title{
New astrometric observations of Phobos with the SRC on Mars Express ${ }^{\star}$
}

\author{
K. Willner, J. Oberst, M. Wählisch, K.-D. Matz, H. Hoffmann, T. Roatsch, R. Jaumann, and V. Mertens \\ German Aerospace Center, Institute of Planetary Research, Planetary Geodesy, Rutherfordstrasse 2, 12489 Berlin, Germany \\ e-mail: konrad.willner@dlr.de \\ Received 17 March 2008 / Accepted 20 May 2008
}

ABSTRACT

\begin{abstract}
Aims. New astrometric measurements for Phobos are reported on the basis of 69 SRC (Super Resolution Channel) images obtained during 28 Mars Express Phobos flybys executed between 2004 and 2007.

Methods. The measurements have been made using a newly developed technique that involves positional measurements of surface control points and verification of camera pointing by background stars.

Results. The astrometric positions are in excellent agreement with currently available Phobos orbit models. However, we find remaining systematic offsets of $1.5-2.6 \mathrm{~km}$ such that Phobos is ahead of its predicted position along the track.

Conclusions. Our observations will be a basis for further improvements in the Phobos ephemeris. The methods that we have developed will be useful for the astrometric tracking of planetary or asteroidal targets and spacecraft optical navigation in future planetary missions.
\end{abstract}

Key words. astrometry - ephemerides - planets and satellites: individual: Phobos

\section{Introduction}

Ever since the discovery of Phobos in 1877 , there has been great interest in the motion of this natural satellite deep in the gravity field of Mars. Since its orbit is affected over long time periods by tidal forces, it can provide us with information about the interior elasticity and thermal evolution of Mars. Depending on the accuracy of the measured data, we can also constrain the gravitational moments of Phobos and seasonal changes in the gravity field parameters of Mars (Lainey et al. 2007; Lainey 2007). The orbit of Phobos has been studied by means of observations from the ground (see Morley 1989; Oberst et al. 2006, and references therein) as well as from the Mariner 9 (Duxbury \& Callahan 1989a), Viking (Duxbury \& Callahan 1988), and Phobos (Kolyuka et al. 1991) spacecraft. More recent observations include those of Mars Global Surveyor (Banerdt \& Neumann 1999), MRO (unpublished data), and those of Mars Express, which were the subject of an earlier paper by our team (Oberst et al. 2006). In addition, observations of Phobos transits across the solar disk have been carried out by the Mars Exploration Rovers (Bell et al. 2005).

We report on astrometric observations of Phobos derived from Mars Express image data. This paper marks a step forward over our previous work. We analyzed four times as many SRC images corresponding to almost two and a half years of observations. We have developed more advanced methods to determine the position of Phobos in the images on the basis of surface control points. In comparison with our previous analysis, we apply a revised version of the SRC planning software which provides more flexibility in the camera pointing during Phobos flybys to capture background stars. Background star observations can be analyzed to control the camera pointing, which was a source of uncertainty in our previous paper.

* Table A.2 is only available in electronic form at the CDS via anonymous ftp to cdsarc.u-strasbg. fr $(130.79 .128 .5)$ or via http://cdsweb.u-strasbg.fr/cgi-bin/qcat?J/A+A/488/361
Motivated by the availability of the early Phobos observations, two groups, one at the Jet Propulsion Laboratory, JPL (Jacobson \& Rush 2006; Jacobson 2007), the other at the Royal Observatory of Belgium, ROB (Lainey et al. 2007), developed revised orbit models of Phobos. On the basis of our astrometric measurements we therefor compare the observed Phobos positions with the two available orbit prediction models.

\section{Measurements}

\subsection{SRC Camera and Phobos flybys}

Mars Express is equipped with the HRSC (High Resolution Stereo Camera), a wide-angle push-broom scanner for the mapping of Mars in 3D (Neukum \& the HRSC Co-I-Team 2005; Jaumann et al. 2007) and the SRC (Super Resolution Channel), a framing camera of large focal length, designed to show details within the HRSC scenes (see Oberst et al. 2006; Oberst et al. 2008, for a camera description and technical parameters). Although both cameras are operated by the same digital unit, they act practically as separate cameras.

The almost polar orbit of Mars Express is highly elliptical (apoapsis altitude of currently 10, $119 \mathrm{~km}$, Jaumann et al. 2007) and (unlike any US spacecraft currently orbiting Mars) reaches well beyond the nearly circular equatorial orbit of Phobos with a distance of $9375 \mathrm{~km}$ from the center of Mars. Since the orbital periods of Mars Express and Phobos are similar, there are typically multiple flybys in consecutive orbits, usually followed by epochs of few or no Phobos encounters (Oberst et al. 2006; Oberst et al. 2008). As of November 2007, Mars Express has engaged in 46 flybys of Phobos at ranges $<3000 \mathrm{~km}$ (see Table A.1), and Phobos or parts of its surface were captured in more than 230 SRC images.

\subsection{Pointing verification}

During a Phobos flyby, the SRC is pointed at some fixed inertial position in the celestial sphere. A sequence of usually 8 images 
Table 1. Comparison of the Hipparcos (Perryman et al. 1997) and the PPM (Röser et al. 1994) star catalogues.

\begin{tabular}{|c|c|c|}
\hline Catalogue & $\begin{array}{l}\text { Hipparcos } \\
\end{array}$ & $\overline{\mathrm{PPM}}$ \\
\hline No. of stars & 118218 & 468586 \\
\hline Stars/deg 2 & $\sim 3$ & $\sim 7.8$ \\
\hline Mean err. position & $0.77 / 0.64^{a}$ & $0.11 / 0.27^{b}$ \\
\hline Visual Mag. & 9.0 & 13.5 \\
\hline
\end{tabular}

${ }^{a} \sigma_{\alpha} \sigma_{\delta}$ in milliarcsec, at $\mathrm{J} 1991.25 ;{ }^{b} \sigma$ in arcsec, dependend on the hemisphere, at J1990.

is executed as Phobos enters the field of view (cf. Oberst et al. 2008). There is typically some freedom in the choice of this inertial pointing vector (within the plane containing Phobos and the spacecraft flyby trajectory) to cover specific Phobos surface areas or to capture background stars. Indeed, the SRC operation software was upgraded to take full advantage of this flexibility. As a consequence, background star observations are available for all flyby image sequences since mid 2005, which can be used to verify camera pointing.

The image coordinates of the background stars are measured and compared with predictions, computed from the nominal camera pointing data and the stellar coordinates from the Hipparcos (ESA 1997) and the PPM (Röser \& Bastian 1992) star catalogs. While the Hipparcos catalogue consists of 118218 star entries with an overall higher accuracy than the PPM catalogue (Perryman et al. 1997), the latter has a higher number of star position entries (Röser et al. 1994) with a wider range of visual magnitudes (cf. Table 1). Visual comparison of star position entries in both catalogues for a given star implied that positional differences between the two star catalogues are less than the measurement accuracy of one SRC pixel.

The nominal pointing data were updated accordingly. In most cases, only one star is visible in the images. Pointing data were adjusted by a shift of the image in the line - and sample direction, assuming that image rotation could be neglected (an assumption that was later verified by the Phobos orientation in the images).

Mostly the faint stars (visual magnitude $+4<m<+10$ ) are captured in SRC's narrow field of view, which require dedicated long-exposure $(516 \mathrm{~ms})$ images. Long exposures are taken typically before and after the Phobos encounter and allow us to check for possible pointing drift during the flyby. The reduction of the background star observations show that the nominal pointing data are on average correct to 25 pixels, while a maximum offset of 45 pixels was observed and 3 gross outliers were found in orbits 682,2706 , and 2739, where observed and predicted image coordinates differed by up to 200 pixels. The relative pointing during one flyby, on the other hand, was found to be very stable with an average pixel offset of 3 pixels between the star observation in the first and the last image of a flyby. Again, one outlier was found (orbit 3245), where no drift was registered in the nominal pointing but star positions in the images differed by 44 pixel.

\subsection{COM measurements}

Much effort was made to improve the method of determining the line/sample coordinates of the Phobos center of mass (COM) in the images. While a fitting of ellipsoid models to the observed limb was used in our previous analysis, we now use positional measurements of surface features.

The control point network by Duxbury \& Callahan (1989b) and Duxbury (1991) was used as a basis. The network involves the 3D Cartesian coordinates of 315 points, exclusively craters

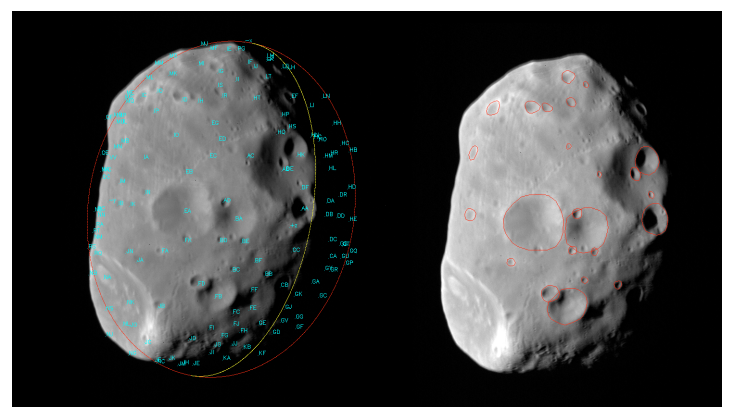

Fig. 1. Left - Phobos with fitted limb (red) and terminator (yellow), as well as control points from the Duxbury and Callahan (Duxbury \& Callahan 1989b; Duxbury 1991) network. The control point identifiers are projected onto the SRC image to rule out miss identifications. Right - fitted ellipses of identified and clearly visible control points.

that were easily identified in images and associated with particular control points in images (see Fig. 1), the coordinates of which, with the respect to the COM, are believed to be known. We note that any differences between COM and COF (center of figure) should be small.

An effort was made to maintain consistent positional measurements for the craters, seen from different ranges and under different viewing angles. The crater rims were mapped (Fig. 1, right), and an ellipse fit routine was used to determine the image coordinates, $x_{\mathrm{m}}, y_{\mathrm{m}}$. On average 9 points (minimum: 3 ) were identified in each image. We note that the control points are defined as the centers of the local mean surface of a crater (not the crater floors).

For each observed control point, we calculated the predicted image coordinates, $x_{\mathrm{p}}, y_{\mathrm{p}}$, using the Phobos orbit model and the corrected camera pointing. These predicted image coordinates, $x_{\mathrm{p}}, y_{\mathrm{p}}$, were then transformed to fit the measured image coordinates, $x_{\mathrm{m}}, y_{\mathrm{m}}$, using the functional model:

$\left[\begin{array}{l}x_{\mathrm{m}} \\ y_{\mathrm{m}}\end{array}\right]=s[R(\alpha)]\left[\begin{array}{l}x_{\mathrm{p}} \\ y_{\mathrm{p}}\end{array}\right]+\left[\begin{array}{l}x_{\text {trans }} \\ y_{\text {trans }}\end{array}\right]$

where the free parameters, rotation $R(\alpha)$, scale $s$, and translation, $x_{\text {trans }}, y_{\text {trans }}$, including their errors, were determined using an iterative least-squares analysis involving all identified surface features of one image at a time. The analysis converged rapidly after only 3 to 4 iterations. Furthermore, it indicated that the scale and rotation parameters could be neglected in all cases, implying that the apparent size and orientation of Phobos were correct to first order.

In a similar way, the Phobos COM (at the origin of the Duxbury and Callahan coordinate system) was converted to predicted image space coordinates. The transformation parameters from above were used to calculate the corrected image position and, finally, the astrometric position of the COM of Phobos.

While over 120 images were available, we analyzed 69 images acquired during 28 flybys in which background star observations were available before and after the Phobos encounter. Images with too limited coverage of Phobos (for which less than 3 control points were identifiable) were not considered (see Fig. 2 for examples).

\section{Results}

Thus, we completed a total of 69 Phobos positional measurements. The error in the measurements was modeled as the sum of accuracies of the determined transformation parameters $\sigma_{\mathrm{t}}$, 


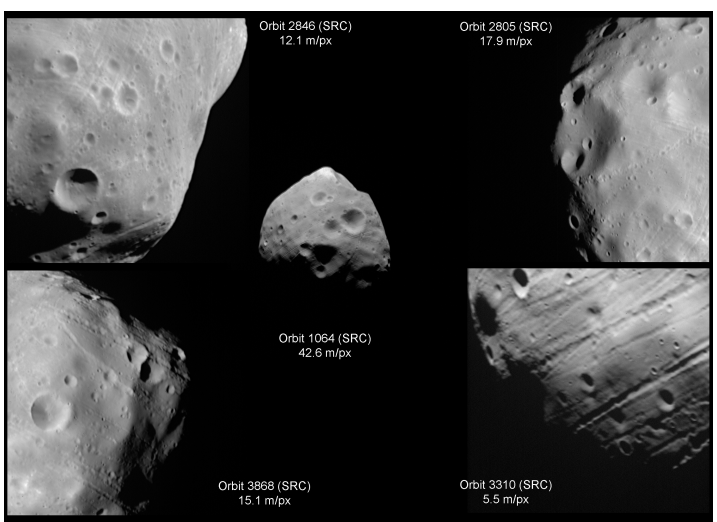

Fig. 2. Examples of SRC images obtained during different flybys. The example from the orbit 3310 (lower right corner) could not be used for astrometric measurements since the scene covers less than 3 known surface features.

the camera pointing $\sigma_{\mathrm{p}}$, and a term that is related to the spacecraft position $\sigma_{\mathrm{sc}}$ and range $\sigma_{\mathrm{r}}$.

$\sigma=\sqrt{\sigma_{\mathrm{t}}^{2}+\sigma_{\mathrm{p}}^{2}+\left(\arctan \frac{\sigma_{\mathrm{sc}}}{r}\right)^{2}}$

Although the positional accuracy of data of Mars Express in its orbit was reported to be of higher quality than $200 \mathrm{~m}$ (Jaumann et al. 2007), for this analysis we used the more conservative previously used value of $\sigma_{\mathrm{sc}}=500 \mathrm{~m}$ (Oberst et al. 2006). The spacecraft range $r$ was computed and is recorded in Table A.1 for each individual flyby. The values $\sigma_{\mathrm{t}}$, and $\sigma_{\mathrm{p}}$, were determined for each individual image. Hence, we estimated the total error to be approximately \pm 0.002 to \pm 0.035 degrees, which translates into Phobos positional errors in the object space of $\pm 0.1 \mathrm{~km}$ to $\pm 0.5 \mathrm{~km}$, perpendicular to the line of sight. For a full list of results, see Table A.2, where astrometric stellar coordinates of Phobos are given with respect to the spacecraft position, and the coordinates of the spacecraft positions in the Mars centered J2000 frame.

\section{Comparison of the orbit models}

The astrometric positions were compared with two Phobos orbit models by Jacobson \& Rush (2006) and Lainey et al. (2007). These are termed the ROB-model (Royal Observatory of Belgium) and JPL-model (Jet Propulsion Laboratory, Solar System Dynamics Group) in the following. According to Jacobson \& Rush (2006), the JPL orbit model used a more up-todate gravitational field representation of Mars as well as the latest planetary constants. The JPL-model was also constrained by the recent Phobos observations by MRO (Mars Reconnaissance Orbiter). The two orbit models differ in the weighing schemes applied to the different observations (cf. Table 2).

While the offsets between the orbit models and observations are small and within the reported nominal errors of the models, the offset vectors appear to be consistent. In particular, it appears that Phobos is ahead of its predicted position by an average of $1.5 \mathrm{~km}$, if compared with the ROB-model and $2.6 \mathrm{~km}$ in comparison with the JPL-model (Table 3 and Fig. 3). There is no significant offset in the across-track direction (out of orbit plane and in a radial direction from the center of the orbit ellipse) of Phobos. We searched for periodic variations in the offsets with Phobos' orbit phase. However, no such systematic effect was found.
Table 2. Differences between the ROB-model (Lainey et al. 2007) and the JPL-model (Jacobson \& Rush 2006).

\begin{tabular}{|c|c|c|}
\hline $\begin{array}{l}\text { Orbit model } \\
\text { Criteria }\end{array}$ & ROB-model & JPL-model \\
\hline $\begin{array}{l}\text { Mars } \\
\text { Gravitational } \\
\text { field }\end{array}$ & $\begin{array}{l}\text { MGM1041C up to } \\
\text { degree } 10\end{array}$ & $\begin{array}{l}\text { MGS95J (Konopliv et al. } \\
\text { 2006) up to degree } 8 \\
\text { for zonal harmonics and } \\
\text { degree and order } 5 \text { for } \\
\text { tesseral harmonics }\end{array}$ \\
\hline $\begin{array}{l}\text { Planetary } \\
\text { Constants }\end{array}$ & DE406 & DE414 \\
\hline Tides & $\begin{array}{l}\text { Tides for both satel- } \\
\text { lites are modelled as } \\
\text { potential }\end{array}$ & $\begin{array}{l}\text { Tides raised by Deimos } \\
\text { are ignored }\end{array}$ \\
\hline $\begin{array}{l}\text { Available } \\
\text { Observations }\end{array}$ & $\begin{array}{l}\text { - HRSC/SRC } \\
\text { - No MRO } \\
\text { - No MGS MOLA } \\
\text { - all previous obser- } \\
\quad \text { vations }\end{array}$ & $\begin{array}{l}\text { - HRSC/SRC } \\
\text { - MRO data } \\
\text { - MGS MOLA } \\
\text { - earthbased observa- } \\
\text { tions from Table } \\
\text { Mountain Observato- } \\
\text { ry } \\
\text { - all previous observa- } \\
\text { tions }\end{array}$ \\
\hline
\end{tabular}

Table 3. Comparison of orbit models with flyby observations. Positive along-track offsets indicate that Phobos is ahead of its predicted position.

\begin{tabular}{ccc}
\hline \hline Orbit Model & Along-track offsets & Across-track offsets \\
\hline ROB-model & $+1.5 \mathrm{~km}$ & $\pm 0.3 \mathrm{~km}$ \\
JPL-model & $+2.6 \mathrm{~km}$ & $\pm 0.5 \mathrm{~km}$ \\
\hline
\end{tabular}

Figure 3 indicates the differences between the ROB orbit model as well as the JPL orbit model and astrometric observations in flyby images in along-track direction over time.

\section{Discussion}

We have presented a new batch of astrometric observations of Phobos on the basis of SRC image data. By applying our revised

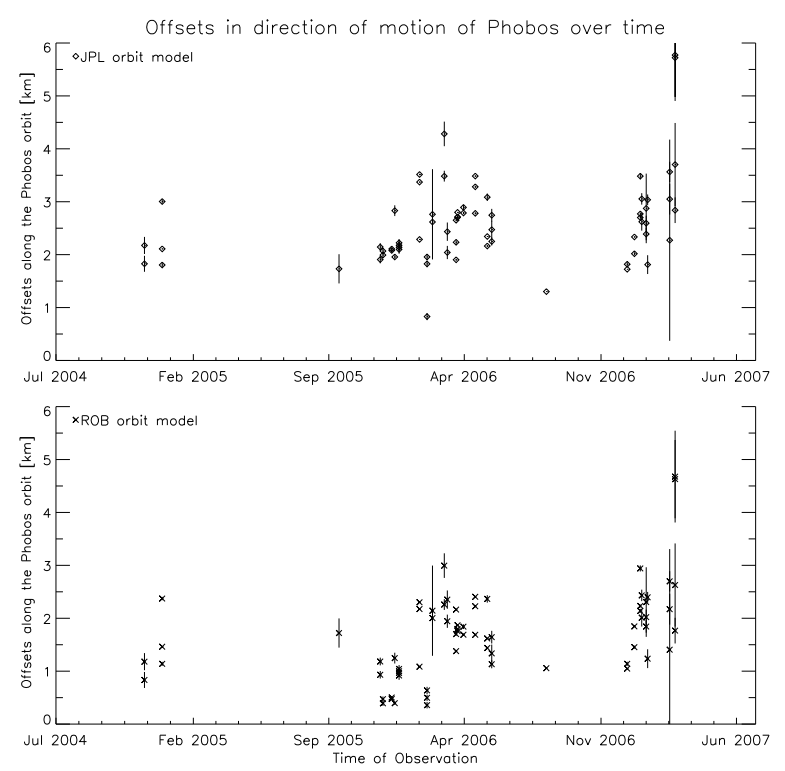

Fig. 3. Differences between ROB and JPL orbit models and SRC observations in along-track direction. Positive numbers show that Phobos is ahead of its position. 
measurement technique and using star observations for pointing control, we have produced accurate observational data that do not suffer significantly from outliers, which were a source of concern in our previous study.

While there was considerable disagreement between previous analysis of Phobos orbit models as well as between the models and astrometric observations (Oberst et al. 2006), there is now widespread consistency between the currently available models (Jacobson \& Rush 2006; Lainey et al. 2007) and good agreement between the models and observations.

There is some remaining concern about the Mars Express trajectory. Could the observed Phobos offset of $1.5 \mathrm{~km}$ to $2.6 \mathrm{~km}$ be caused by a systematic error in the Mars Express orbit calculation? This possibility cannot be entirely excluded. However, since the orbit planes of Phobos and MEX are almost perpendicular, such a systematic offset in the observed Phobos along-track direction would be difficult to construct. Besides, tests in which we shifted the spacecraft orbit by $\sim 1 \mathrm{~km}$ indicated that the spacecraft position had only a minimal influence on the along-track observations and the results were not change significantly.

However, small but noticeable systematic offsets between models and observations remain. Hence, revised Phobos orbit modeling is being developed (Lainey 2007). Our accurate observational data could ensure that new modeling approaches are possible, involving wider parameter space to yet unknown frontiers. At this stage, astrometric observations over long periods and orbit modeling are the only possible way to estimate the gravitational moments or librations of Phobos, which would otherwise require data from a Phobos orbiter and/or Phobos lander. Furthermore, with over 130 years of astrometric observations of Phobos of ever increasing accuracy, long-term seasonal variations in the gravitational field of Mars may possibly be revealed.

\section{References}

Banerdt, W. B., \& Neumann, G. A. 1999, in Lunar and Planetary Institute Conference Abstracts, 2021

Bell, J. F., Lemmon, M. T., Duxbury, T. C., et al. 2005, Nature, 436, 55

Duxbury, T. C. 1991, Planet. Space Sci., 39, 355

Duxbury, T. C., \& Callahan, J. D. 1988, A\&A, 201, 169

Duxbury, T. C., \& Callahan, J. D. 1989a, A\&A, 216, 284

Duxbury, T. C., \& Callahan, J. D. 1989b, Icarus, 77, 275

ESA 1997, VizieR Online Data Catalog, 1239, 0

Jacobson, R. A. 2007, in First International Conference on the Exploration of Phobos and Deimos, Proceedings of the conference held November 5-8, 2007 in Moffett Field, California, LPI Contribution, 1377, 21

Jacobson, R. A., \& Rush, B. 2006, Ephemerides of the Martian Satellites MAR063, JPL IOM 343R-06-004

Jaumann, R., Neukum, G., Behnke, T., et al. 2007, Planet. Space Sci., 55, 928

\section{Appendix A: Mars Express' Phobos flybys - observations and results}

Table A.1. Mars Express Phobos encounters.

\begin{tabular}{|c|c|c|c|c|}
\hline No. & $\begin{array}{l}\text { MEX } \\
\text { orbit }\end{array}$ & $\begin{array}{c}\text { Encounter time } \\
\text { [UTC] }\end{array}$ & $\begin{array}{r}\text { Phobos true } \\
\text { anomaly } \\
\text { [deg] }\end{array}$ & $\begin{array}{r}\text { Flyby } \\
\text { distance } \\
{[\mathrm{km}]}\end{array}$ \\
\hline 1 & 1064 & 2004-11-16T14:21:50.946 & 120.7 & 4686 \\
\hline 2 & 1163 & 2004-12-14T08:05:08.883 & 101.3 & 3825 \\
\hline 3 & 2151 & 2005-09-16Т23:24:21.222 & 142.0 & 3835 \\
\hline 4 & 2381 & 2005-11-20T09:06:19.796 & 97.0 & 3384 \\
\hline 5 & 2397 & 2005-11-24T20:38:56.692 & 113.3 & 1928 \\
\hline 6 & 2446 & 2005-12-08T13:44:00.307 & 106.0 & 2049 \\
\hline 7 & 2463 & 2005-12-13T08:02:51.150 & 80.8 & 4306 \\
\hline 8 & 2487 & 2005-12-20T01:15:06.755 & 100.1 & 2661 \\
\hline 9 & 2601 & 2006-01-20T23:07:43.213 & 109.4 & 5276 \\
\hline 10 & 2643 & 2006-02-01T17:26:22.171 & 62.7 & 4842 \\
\hline 11 & 2673 & 2006-02-10T04:40:21.065 & 255.0 & 2129 \\
\hline 12 & 2739 & 2006-02-28T16:24:42.868 & 238.5 & 1799 \\
\hline 13 & 2756 & 2006-03-05T11:05:35.566 & 230.6 & 4179 \\
\hline 14 & 2805 & 2006-03-19T04:19:04.148 & 229.8 & 1973 \\
\hline 15 & 2813 & 2006-03-21T09:58:56.585 & 232.9 & 840 \\
\hline 16 & 2846 & 2006-03-30T15:54:53.716 & 227.6 & 1337 \\
\hline 17 & 2912 & 2006-04-18T03:46:10.245 & 216.5 & 2405 \\
\hline 18 & 2979 & 2006-05-06Т23:15:08.280 & 204.1 & 5104 \\
\hline 19 & 3005 & 2006-05-13T23:41:03.708 & 203.3 & 5319 \\
\hline 20 & 3310 & 2006-08-07T10:50:42.982 & 163.9 & 605 \\
\hline 21 & 3761 & 2006-12-11T21:23:53.534 & 277.7 & 2052 \\
\hline 22 & 3802 & 2006-12-23T08:54:31.697 & 271.4 & 891 \\
\hline 23 & 3835 & 2007-01-01T14:51:48.802 & 267.1 & 1253 \\
\hline 24 & 3843 & 2007-01-03T20:30:11.752 & 269.0 & 664 \\
\hline 25 & 3868 & 2007-01-10T20:46:41.277 & 260.9 & 1668 \\
\hline 26 & 3876 & 2007-01-13T02:26:09.255 & 263.7 & 1240 \\
\hline 27 & 3999 & 2007-02-16T13:04:40.907 & 250.1 & 3826 \\
\hline 28 & 4030 & 2007-02-25T02:44:11.994 & 200.7 & 11295 \\
\hline
\end{tabular}

Kolyuka, Y., Tikhonov, V., Ivanov, N., et al. 1991, A\&A, 244, 236

Konopliv, A. S., Yoder, C. F., Standish, E. M., Yuan, D.-N., \& Sjogren, W. L. 2006, Icarus, 182, 23

Lainey, V. 2007, personal communication

Lainey, V., Dehant, V., \& Pätzold, M. 2007, A\&A, 465, 1075

Morley, T. A. 1989, A\&AS, 77, 209

Neukum, G., \& the HRSC Co-I-Team 2005, The HRSC experiment and scientific results, Noordwijk

Oberst, J., Matz, K. D., Roatsch, T., et al. 2006, A\&A, 447, 1145

Oberst, J., Schwarz, G., Behnke, T., Hoffmann, H., et al. 2008, Planet. Space Sci., 56,473

Perryman, M. A. C., Lindegren, L., Kovalevsky, J., et al. 1997, A\&A, 323, L49

Röser, S., \& Bastian, U. 1992, Bulletin d'Information du Centre de Donnees Stellaires, 42, 11

Röser, S., Bastian, U., \& Kuzmin, A. 1994, A\&AS, 105, 301 